\title{
Treatment of severe frostbite with iloprost in northern Canada
}

\author{
Alexander Poole MD, Josianne Gauthier BPharm MScPharm
}

\section{Case 1}

A 46-year-old man was evacuated by air ambulance to Whitehorse General Hospital from the Yukon Arctic Ultra race, a 692-km running race. He had been running for 27 hours in temperatures ranging from $-45^{\circ} \mathrm{C}$ to $-50^{\circ} \mathrm{C}$. Wearing only thin gloves, he had repeatedly exposed his hands to cold air and cold objects to drink, eat and adjust his gear. He had been running on snow, wearing cross-country running shoes. Feeling numbness in his hands and feet, he stopped running and passively rewarmed his hands in his sleeping bag while waiting for rescue. He presented to the emergency department with pain and discomfort in his hands and feet.

On presentation, the patient's core temperature was $36.8^{\circ} \mathrm{C}$. He had evidence of grade 3 frostbite to the right hand, with hemorrhagic blistering into the proximal phalanges of the fourth and fifth digits (Figure 1). His left hand showed evidence of frostbite on the volar aspect of the distal phalanges of the second and fourth digits. He also had frostbite to his left first and second toe, his right foot and his nose. He had no other injuries. He was healthy and athletic, with no contributing medical history. He was a nonsmoker and had not consumed alcohol recently.

The patient's hands and feet were placed in a whirlpool bath of water mixed with chlorhexidine at $38^{\circ} \mathrm{C}$ until they were pink and warm. He was given ibuprofen $600 \mathrm{mg}$ by mouth every six hours and fentanyl and morphine intravenously for pain. Once the patient's hands and feet were rewarmed, the clear blisters were aspirated, after which aloe vera ointment and porous lowadherent wound dressings were applied.

The patient was given iloprost intravenously, titrated to the maximum dose of $2 \mathrm{ng} / \mathrm{kg}$ per min, for six hours daily for five days. The infusion rate was increased gradually to avoid adverse effects (e.g., headaches or hypotension). He tolerated the infusion well.
On follow-up six months later, the patient's hands and feet showed complete healing. Amputation had not been required, but he reported hypersensitivity to touch and temperature.

\section{Case 2}

A 43-year-old man presented to the emergency department of Whitehorse General Hospital after completing the 100-mile (161-km) event of the Yukon Arctic Ultra race. He ran in temperatures ranging from $-30^{\circ} \mathrm{C}$ to $-50^{\circ} \mathrm{C}$ for 64 hours and then rewarmed his feet in a hot tub at his hotel. On presentation, he reported that his toes were numb and white. He was given oral cephalexin and discharged home. He returned to the emergency department 48 hours later concerned about a loss of sensation in his great toes. He recognized that he had frostbite and was seeking an alternative approach to watchful waiting.

The patient had grade 3 frostbite to both great toes, with some mild erythematous extension into the metatarsophalangeal area of his right foot (Figure 2).

The patient was a healthy and athletic orthopedic surgeon, with no contributing medical history. He was a nonsmoker and consumed alcohol occasionally. He was an experienced adventurer and had travelled to both poles in the past.

The oral antibiotic was stopped, and the patient was given tetanus prophylaxis, ibuprofen and topical aloe vera ointment. He also received

\section{KEY POINTS}

- The current standard of care for severe frostbite is rapid rewarming, débridement and aspiration of clear blisters, and application of dry bulky dressings.

- The current approach may be insufficient to prevent necrosis, with amputation often becoming the only solution.

- Studies have shown a decreased amputation rate with use of vasodilators and thrombolytics.

- Recent experience shows that administration of iloprost to treat patients with frostbite is feasible in a community hospital setting. 
one six-hour infusion of iloprost, titrated to a dose of $2 \mathrm{ng} / \mathrm{kg}$ per min, before returning home to Europe. He tolerated the infusion with no adverse reaction. Arrangements were made to continue the five-day course of iloprost infusions in Europe, but the second infusion was missed because the drug was not available at any other North American centre during his transit home. By follow-up at six months, his feet had continued to heal, and he had successfully climbed Mount Olympus in trail shoes. He was unable to kick a ball or undertake ice climbing because of sensitivity at the tips of his toes, but he reported no problems with day-to-day function.

\section{Discussion}

Advancements in the treatment of frostbite have been slow, but studies have shown a decrease in amputation rate with both vasodilators and thrombolytics. ${ }^{1-4}$ The adage of "freezing in the winter and amputating in the spring" no longer holds. However, these advances in treatment have not gained widespread traction in northern Canadian centres, which for geographic reasons are likely to treat numerous patients with exposure to cold. This growing body of evidence should encourage more active treatment of frostbite.

Frostbite occurs when the skin temperature dips below $-2^{\circ} \mathrm{C}$. Cold exposure in the outdoors is generally characterized by slow cooling of the tissues and thus is dominated by extracellular ice formation, rather than the intracellular ice formation that occurs with rapid cooling. The changes observed with advanced frostbite, such as blister formation and friable tissues, are actually postrewarming phenomena, which may continue to develop as much as 72 hours later. ${ }^{5}$ The rewarming phase results in marked cellular injury characterized by the twin processes of vasoconstriction and microthrombosis. ${ }^{5}$ Interventions in the field and in hospital aim to mitigate the sequelae of these events.

Whitehorse is the capital city of Canada's Yukon Territory, located at $60.7^{\circ} \mathrm{N}$ latitude. The frostbite season, when the temperature periodically drops to $-40^{\circ} \mathrm{C}$ or even colder, typically stretches from November to April. An internal retrospective chart analysis at Whitehorse General Hospital for the 10-year period 2005-2014 showed that an average of 14 patients per year presented to the emergency department with frostbite. Over the 10 years reviewed, 25 patients presented with severe frostbite (grade 3 or 4). Six of those advanced cases required amputation. This number may underestimate the per-digit rate of amputation, as the review graded severity on the basis of the most affected digit (i.e., patients with multiple amputations were counted only once).

\section{Diagnosis}

Diagnosis of frostbite is based on a history of cold exposure and the presence of skin changes that are not completely reversed by rewarming.

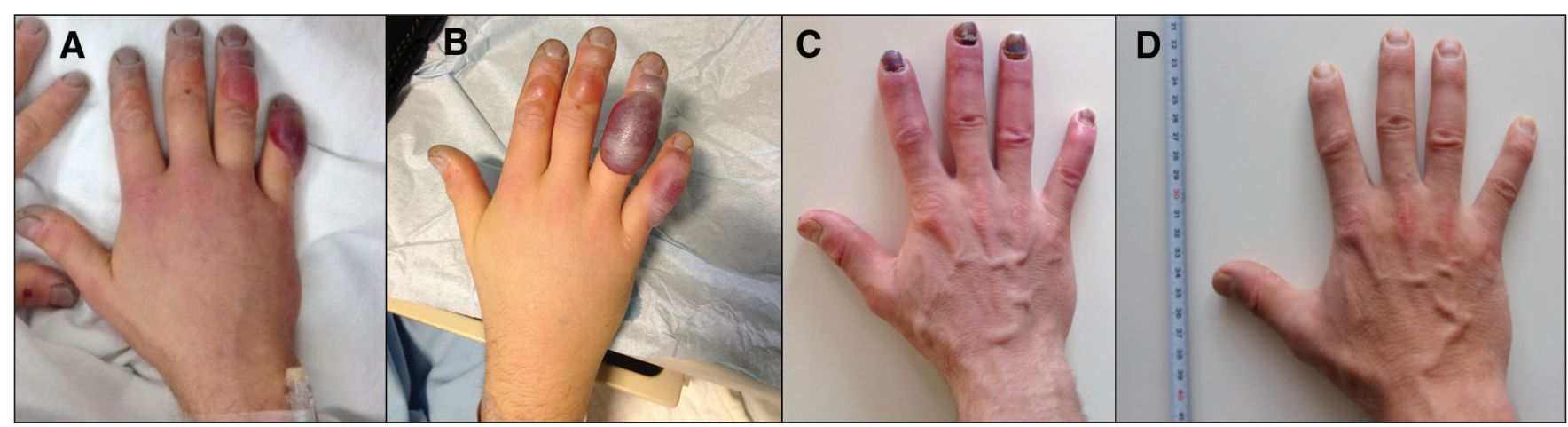

Figure 1: Case 1, a 46-year-old man with grade 3 frostbite on his right hand. (A) Day 1. (B) Day 2. (C) At one month. (D) At six months.
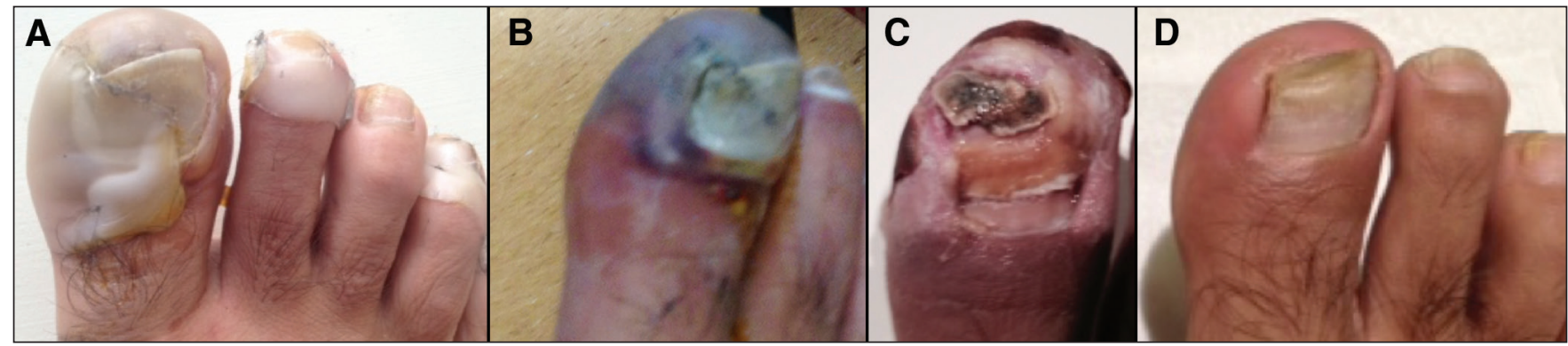

Figure 2: Case 2, a 43-year-old man with grade 3 frostbite on his right foot. (A) Day 1. (B) Day 2. (C) At two months. (D) At six months. 
In frostbite, rewarming - either passively or actively - is accompanied by substantial pain. Frostbite severity can be assessed by the proximal extent of skin changes on the extremities. After rewarming, changes due to frostbite, such as blistering, become more evident but are not immediate. As such, accurate grading of frostbite severity may take up to 48 hours after rewarming. There is evidence that early bone scanning may predict grade at presentation, ${ }^{6}$ but such technology is not available in many northern communities. We use the frostbite severity grading system developed by Cauchy and colleagues ${ }^{6}$ (Box 1 ).

\section{Treatment}

In 2014, in an attempt to modernize our cold injury protocols, we performed a literature review (unpublished) on the treatment of frostbite and consulted with experts in Alaska and Europe. We developed a comprehensive protocol and order set tailored to our community hospital resources and our location in Canada's far north (see Box 2 for a summary; complete protocol given in Appendix 1, available at www.cmaj.ca/lookup/suppl/ doi:10.1503/cmaj.151252/-/DC1). This protocol incorporates three intravenous medications: iloprost, alteplase and heparin. Iloprost is used for grade 3 and 4 cases, whereas alteplase and heparin are used exclusively for grade 4 cases.

At many institutions, the treatment of frostbite has remained stagnant since the last leap forward, the advent of rapid rewarming (described by Mills and associates in 1960). ${ }^{7}$ That work on rewarming established how treatment should begin and led to the current standard of rapid rewarming, débridement and aspiration of clear blisters, and application of dry bulky dressings. ${ }^{8-11}$

The current challenge is to move beyond supportive care in the hospital to treatment that further decreases the amputation rate and long-term sequelae. In severe frostbite (grades 3 and 4), early rapid rewarming and careful tissue management may be augmented by pharmacologic therapy, including topical aloe vera ointment, oral ibuprofen and possibly intravenous therapy. In essence, the current pursuit is to address postrewarming vasospasm and thrombosis. Vasodilators, such as iloprost, and fibrinolytics, such as alteplase, may play a role in mitigating the damage caused to the frostbitten tissues.

\section{Vasoactive agents}

Iloprost is a stable metabolite of prostaglandin $\mathrm{I}_{2}$ (also referred to as prostacyclin). It is a potent vasodilator, inhibits platelet aggregation and enhances fibrinolytic activity. ${ }^{12}$ These characteristics make it a drug of choice to reverse the marked vasoconstriction and microthrombosis occurring in frostbite. Iloprost is also used in Raynaud syndrome, severe peripheral arterial occlusive disease $^{12}$ and advanced thromboangiitis obliterans (Buerger disease). At present, it is not commercially available in an intravenous form in either Canada or the United States. The only drug with similar pharmacologic properties available in Canada is epoprostenol, which is used for pulmonary hypertension; however, to our knowledge, it has never been studied in the context of frostbite.

Iloprost has been used in Europe for severe frostbite since the 1990s, with the first report of its use published in 1994. ${ }^{13}$ Cauchy and colleagues ${ }^{1}$ subsequently described its use in the only prospective randomized controlled trial for frostbite. In that study, iloprost was shown to decrease the risk of amputation relative to control. We requested and received permission to use iloprost through Health Canada's Special Access Programme.

Three small retrospective studies using alteplase in frostbite have been published..$^{2-4}$ Alteplase is a recombinant tissue plasminogen

\begin{tabular}{|lll|}
\hline \multicolumn{3}{|l|}{ Box 1: Grading of frostbite of the extremities } \\
\hline Grade & Proximal extension & \multicolumn{1}{c|}{ Skin changes } \\
\hline 1 & Distal phalanx & Absence of blisters \\
\hline 2 & Middle phalanx & Clear blisters \\
\hline 3 & Proximal phalanx & Hemorrhagic blisters \\
\hline 4 & Metacarpal or tarsal & Hemorrhagic blisters \\
\hline
\end{tabular}

Box 2: Summary of the Whitehorse General Hospital (Yukon Hospital Corporation) frostbite protocol*

- Surgical consultation

- Rapid rewarming of the affected digits in hot water $\left(39^{\circ} \mathrm{C}\right)$ with chlorhexidine and isopropyl alcohol

- Immersion of affected parts in hot water $\left(39^{\circ} \mathrm{C}\right)$ in a hydrotherapy whirlpool daily (starting the day after rewarming)

- Débridement and aspiration of clear blisters

- Application of aloe vera protective ointment and porous low-adherent wound dressings

- Elevation of affected parts

- Avoidance of tobacco and alcohol

- Tetanus-diphtheria immunization

- Oral ibuprofen every 6 hours

For grade 3 or higher frostbite:

- Intravenous infusion of iloprost $2 \mathrm{ng} / \mathrm{kg}$ per $\min , 6 \mathrm{~h} / \mathrm{d}$, for 5 days

For grade 4 frostbite:

- After administration of iloprost, concurrent intravenous infusion of alteplase (for one day; weight-based dosage, progressively increased to a maximum total dose of $100 \mathrm{mg}$ ) and heparin (for 72 hours; dosage based on weight and prothrombin time)

*The complete protocol and dosing information are available in Appendix 1 (www.cmaj.ca/ lookup/suppl/doi:10.1503/cmaj.151252/-/DC1). 
activator. With its thrombolytic properties, it reverses the small-vessel thrombosis that occurs in frostbite and helps to restore perfusion to frostbitten digits. ${ }^{12}$ In the published studies, ${ }^{2-4}$ alteplase was associated with a decrease in the rate of amputation of severely frostbitten digits. Administration of the vasodilator iloprost before initiation of alteplase is thought to have an additive effect, but this has yet to be demonstrated in trials. ${ }^{1}$ In all studies using alteplase, heparin infusion has also been administered.

\section{Conclusion}

Our recent experience shows that treating frostbite with iloprost is feasible in a community hospital setting. This approach may have contributed to healing of the extremities without the need for amputation. Our development of a comprehensive protocol based on current evidence could eventually lead to a concerted approach that may be suitable for other northern community hospitals where frostbite is treated.

\section{References}

1. Cauchy E, Cheguillaume B, Chetaille E. A controlled trial of a prostacyclin and rt-PA in the treatment of severe frostbite. N Engl J Med 2011;364:189-90.

2. Twomey JA, Peltier GL, Zera RT. An open-label study to evaluate the safety and efficacy of tissue plasminogen activator in treatment of severe frostbite. J Trauma 2005;59:1350-4.

3. Bruen KJ, Ballard JR, Morris SE, et al. Reduction of the incidence of amputation in frostbite injury with thrombolytic therapy. Arch Surg 2007; 142:546-51.

4. Johnson AR, Jensen HL, Peltier G, et al. Efficacy of intravenous tissue plasminogen activator in frostbite patients and presentation of a treatment protocol for frostbite patients. Foot Ankle Spec 2011;4:344-8.

5. Mohr WJ, Jenabzadeh K, Ahrenholz DH. Cold injury. Hand Clin 2009;25:481-96

6. Cauchy E, Chetaille E, Marchand V, et al. Retrospective study of 70 cases of severe frostbite lesions: a proposed new classification scheme. Wilderness Environ Med 2001;12:248-55.

7. Mills WJ, Whaley R, Fish W. Frostbite, I: experience with rapid rewarming and ultrasonic therapy. Alaska Med 1960;2:1-4.

8. Hallam MJ, Cubison T, Dheansa B, et al. Managing frostbite. BMJ 2010;341:c5864.

9. McIntosh SE, Hamonko M, Freer L, et al. Wilderness Medical Society practice guidelines for the prevention and treatment of frostbite. Wilderness Environ Med 2011;22:156-66.
10. Zafren K. Frostbite: prevention and initial management. High Alt Med Biol 2013;14:9-12.

11. Handford C, Buxton P, Russell K, et al. Frostbite: a practical approach to hospital management. Extrem Physiol Med 2014;3: 7-10.

12. Musiał J, Wilczynska M, Sladek K, et al. Fibrinolytic activity of prostacyclin and iloprost in patients with peripheral arterial disease. Prostaglandins 1986;31:61-70.

13. Groechenig E. Treatment of frostbite with iloprost. Lancet 1994; 344:1152-3.

Affiliations: Whitehorse General Hospital (Poole, Gauthier), Yukon Hospital Corporation, Whitehorse, Yukon; Department of Surgery (Poole), Cumming School of Medicine, University of Calgary, Calgary, Alta.; College of Pharmacy and Nutrition (Gauthier), University of Saskatchewan, Saskatoon, Sask.

Contributors: Alexander Poole was the attending surgeon for the patients described here, and Josianne Gauthier offered clinical pharmacy consultation on the cases. Alexander Poole conducted the literature and chart review, and contacted specialists in Europe and Alaska. Josianne Gauthier conducted the literature review and contacted specialists in Europe. Both of the authors developed the frostbite protocol, drafted and revised the article, approved the final version to be published and agreed to act as guarantors of the work.

Funding: The authors did not receive funding for this article and did not receive any other form of compensation to use the drugs mentioned in this article. The drugs were provided by the hospital pharmacy department for inpatient use; iloprost was obtained at no cost through Health Canada's Special Access Programme.

Acknowledgements: The authors would like to thank Dr. Ken Zafren for encouragement to publish their experience and for commenting on a draft version; Dr. James E. O'Malley for sharing his experience with frostbite cases in Alaska; and Dr. Emmanuel Cauchy for sharing his protocol, as well as his experience using iloprost.

The section Cases presents brief case reports that convey clear, practical lessons. Preference is given to common presentations of important rare conditions, and important unusual presentations of common problems. Articles start with a case presentation (500 words maximum), and a discussion of the underlying condition follows (1000 words maximum). Visual elements (e.g., tables of the differential diagnosis, clinical features or diagnostic approach) are encouraged. Consent from patients for publication of their story is a necessity. See information for authors at www.cmaj.ca. 